\title{
Risk-based oral cancer screening - lessons to be learnt
}

\author{
Anil K.D’Cruz (1) and Richa Vaish
}

\author{
The Kerala Oral Cancer Screening Trial did not demonstrate an overall \\ cancer-related mortality benefit. Herein, we discuss the important lessons \\ learnt from a recent reanalysis of data from this trial in an attempt to \\ demonstrate the advantages of using a novel risk-based approach to cancer \\ screening.
}

Refers to Cheung L. C. et al. Risk-based selection of individuals for oral cancer screening. J Clin. Oncol. 39, 663-674 (2021).

The basic premise of screening is to detect cancers early in order to improve survival, administer less-intensive treatments and decrease morbidity. The success story of screening for cervical cancers has been well established. Screening recommendations are also in place in many countries for breast, colon, lung and prostate cancers ${ }^{1}$. Screening has benefits but also limitations, the most important of which are its associated costs and barriers to accessibility even in the most advanced health-care systems. Now, the reanalysis of the previously published Kerala Oral Cancer Screening Trial (KOCST) provides interesting lessons to learn from ${ }^{2}$.

Oral cancers are a global problem, with a mortality rate of $\sim 50 \%^{3}$. Despite the ease of access to examination, these cancers present late with disastrous outcomes on survival and quality of life. Two-thirds of cancers and three-quarters of cancer-related deaths occur in $\mathrm{Asia}^{3}$ and, thus, this region would benefit from implementing screening programmes. Prior attempts have focused on increasing detection rates of premalignant lesions and cancers using oral examination or adjuncts such as vital staining, cytology and light-based techniques ${ }^{4}$. To date, the KOCST is the only trial that has reduction in mortality as the end point ${ }^{5}$, an essential prerequisite to establish benefit from any screening programme. This randomized controlled trial consisted of three rounds of screening at 3-yearly intervals in which health-care workers performed visual oral examination. Individuals in the control group underwent health awareness education. With a mortality rate ratio of
0.79 (95\% CI 0.51-1.22), the difference was not statistically significant across the entire cohort; however, a statistically significant reduction was observed in the high-risk group (HRG), comprising ever tobacco and/or alcohol users, with a mortality rate ratio of 0.66 (95\% CI $0.45-0.95)^{6}$. Screening was performed at $<$ US $\$ 6$ per person and deemed to be cost-effective, particularly in the HRG. The incremental cost per life-year saved was $\$ 835$ and $\$ 156$ for the entire cohort and HRG, respectively ${ }^{7}$. Despite these tangible benefits, this study had limitations in methodology ${ }^{2,4}$ and, thus, the results were not considered robust enough to support incorporation into national screening programmes.

In the recent reanalysis of the KOCST, researchers evaluated a novel risk-based screening strategy that could help direct efforts at those most likely to benefit ${ }^{2}$. The relative and absolute efficacy of screening was estimated across the entire cohort, amongst the HRG and across quartiles of a 7-year oral-cancer risk-prediction model including covariates chosen a priori (age, sex, education, body mass index, duration and intensity of tobacco chewing, smoking and alcohol use, interaction between chewing duration and smoking intensity, and study arm). The results confirmed that screening is most effective in the HRG, with a correlation between increasing efficacy and model-predicted risk (from the lowest to the highest quartile). Translated into numbers, 2,043 individuals would need to be screened to prevent one death from oral cancer across the entire population. The efficiency of the screening programme would be scarce resources could ... be judiciously used targeting individuals most likely to benefit

improved with targeted screening of individuals in the HRG and even further enhanced with risk-prediction model-based screening of the $50 \%$ of individuals at the highest risk, reducing the number that need to be screened to prevent one oral cancer-related death to 1,029 and 610 , respectively. These findings provide proof-of-principle that risk-based screening could have substantial benefits.

This approach would be particularly appropriate in resource-constrained countries with a high incidence of oral cancer, many with a lack of basic infrastructure and existing screening programmes. Scarce resources could then be judiciously used targeting individuals most likely to benefit. Even in high-income countries, where oral cancer screening is not accorded high priority owing to its low prevalence, this approach would be conceptually attractive. Some concerns remain, however. Individuals in the HRG, prone to substance abuse, are the least likely to comply with screening. Indeed, only $20 \%$ of eligible population completed the planned screening visits in the $\mathrm{KOCST}^{8}$, a high literacy rate ( $>90 \%$ ) notwithstanding 9 . In addition, nearly one-third of cancers diagnosed in the intervention arm were detected as interval cancers ${ }^{6}$. Owing to the high incidence of interval cancers, this biologically aggressive cancer should logically be screened more frequently than every 3 years. Such shorter intervals would additionally erode the number of individuals that undergo screening and also increase costs incurred. Furthermore, in contrast to cervical cancers, oral cancers do not have as welldefined a tumour progression model. Not all cancers can be traced back to arise from premalignant lesions, and only $5 \%$ of such lesions progress to cancer. In the absence of proven molecular tests to identify high-risk premalignant lesions, many asymptomatic individuals would undergo unwanted procedures, placing additional burden on the health-care system as well as subjecting individuals to unnecessary anxiety and morbidity.

These concerns suggest that the inclusion of routine oral cancer screening will not 
become a reality in the immediate future until more robust data emerge. However, we can learn important lessons from this reanalysis. Targeting the HRG holds promise as a resource-efficient alternative, a concept that can be extrapolated to screening of other cancers as well. Moreover, tobacco and/or alcohol use places individuals at an increased risk of other non-communicable diseases (NCD). This approach offers the advantage of potentially targeting multiple NCDs in addition to other aerodigestive tract cancers, given their common aetiological factors, enabling overlapping health-care parameters to be checked along with providing education on lifestyle and preventive aspects.

A similar risk-based approach has been investigated to triage individuals for breast cancer screening, given restrictions owing to the ongoing COVID-19 pandemic. In this study, patients were stratified into subgroups according to breast-cancer detection rates ${ }^{10}$. The $12 \%$ of mammograms performed in individuals at very high or high risk (89.6 and 36.1 cancers detected per 1,000 mammograms, respectively) accounted for $55 \%$ of tumours.
A non-risk-based approach resulted in the detection of 11.5 cancers per 1,000 mammograms. Risk-based approaches would make perfect sense in the current pandemic, given that they can be focused on populations most likely to benefit while protecting others from unnecessary exposure at health-care facilities. In summary, risk-based screening seems a logical way forward to increase cancer-related survival in the immediate future until improvements in health-care systems and screening programmes enable broader population coverage worldwide.

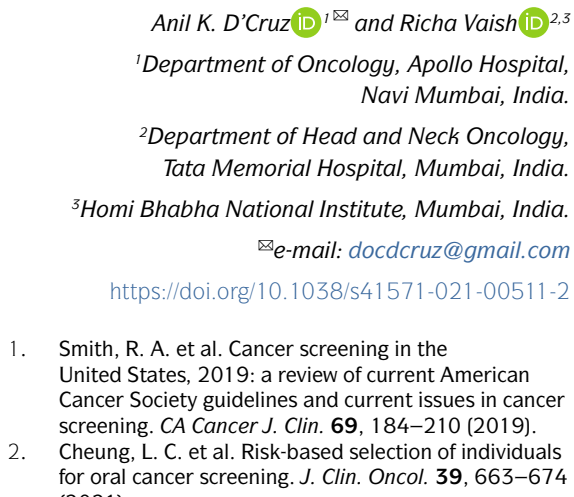

2. Cheung, L. C. et al. Risk-based selection of individuals for oral cancer screening. J. Clin. Oncol. 39, 663-674 (2021).
3. International Agency for Research on Cancer. Global Cancer Observatory: Cancer Today. iarc.fr https:/ gco.iarc.fr/today (2021).

4. Brocklehurst, P. et al. Screening programmes for the early detection and prevention of oral cancer. Cochrane Database Syst. Rev https:/ doi.org/10.1002/14651858.CD004150.pub2 (2013).

5. Sankaranarayanan, R. et al. Early findings from a community-based, cluster-randomized, controlled oral cancer screening trial in Kerala, India. The Trivandrum Oral Cancer Screening Study Group. Cancer 88, 664-673 (2000).

6. Sankaranarayanan, R. et al. Trivandrum Oral Cance Screening Study Group. Effect of screening on oral cancer mortality in Kerala, India: a cluster-randomised controlled trial. Lancet 365, 1927-1933 (2005).

7. Subramanian, S. et al. Cost-effectiveness of oral cancer screening: results from a cluster randomized controlled trial in India. Bull. World Health Organ. 87 200-206 (2009).

8. Sankaranarayanan, R. et al. Long term effect of visual screening on oral cancer incidence and mortality in a randomized trial in Kerala, India. Oral Oncol. 49, 314-321 (2013).

9. India Today. Kerala most literate state at $96.2 \%$ in new report, Andhra, Rajasthan worst performers. indiatoday.in https://www.indiatoday.in/india/story/ kerala-most-literate-state-at-96-2-in-new-reportandhra-rajasthan-worst-performers-1719614-202009-08 (2020)

10. Miglioretti, D. L. et al. Assessment of a risk-based approach for triaging mammography examinations during periods of reduced capacity. JAMA Netw. Open. 4, e211974 (2021)

\section{Competing interests}

The authors declare no competing interests. 